\title{
Enhancing Mobility Based on Realistic Models for Vehicular Ad Hoc Networks
}

\author{
V. Hariharasudhan, P. Vetrivelan
}

\begin{abstract}
Creation and maintaining of the one-to-one communication link between the nodes in Vehicular Adhoc Networks are challenging. The model which is very much efficient for one particular scenario will not work at the same level of efficiency for another environment. The mobility of the nodes in the network plays a crucial role in establishing a reliable communication model in the VANETs. Analyzing the nature of mobility inside a particular network based on logical and historical data paves an efficient way in the routing of packets by predicting the best route and improve the quality of the network, reliability, and other performance in terms of serviceability. This paper aims to analyze the drawbacks of existing mobility models utilizing various network quality parameters by classifying them into microscopic and macroscopic mobility models. With the insight gained from the analysis, we propose two methodologies where the realistic model for the VANETs can be established. The models are architected with the help of the information provided by the Geographic information system. The conventional mobility models include excessive details such as road and street layouts, intersection with traffic signals, acceleration and deceleration, building, and other obstacles in a realistic mobility model, that requires prolonged time to design and optimize, it should complicate the simulation. Designing an effective, realistic mobility model is crucial. The key objectives of this proposal are to architect realistic VANETs mobility models by taking into account the real-time road environment and actual data according to the traffic demand and improving the real-time performance of VANETs.
\end{abstract}

Keywords: ITS, Mobility models, VANETs, Routing, Internet of Vehicles, clustering, mobility metric, spatial dependency.

\section{INTRODUCTION}

\section{T} he Vehicular Adhoc NETworks (VANETs) comprises a cluster of mobile vehicles that can communicate between them with the use of a fixed infrastructure, named as Road Side Units (RSUs) [1]. The VANETs inter-communication has recently emerged as a fascinating research topic in the development of the Intelligent Transportation System (ITS). The ITS aims at developing communication protocols for VANETs to improve driving safety, infotainment, and traffic efficiency. To analyze the performance of the protocols and

Manuscript received on April 20, 2021.

Revised Manuscript received on April 27, 2021.

Manuscript published on April 30, 2021.

* Correspondence Author

Hariharasudhan $\mathbf{V}^{*}$, SENSE, VIT University, Vellore, India. Email: hariharasudhan.v@vit.ac.in

Vetrivelan P, SENSE, VIT University, Vellore, India. Email: vertivelan.p@vit.ac.in

(c) The Authors. Published by Blue Eyes Intelligence Engineering and Sciences Publication (BEIESP). This is an open access article under the CC BY-NC-ND license (http://creativecommons.org/licenses/by-nc-nd/4.0/) architecture used in the VANETs, the simulation of the network parameters is an effective methodology. Even though real-world deployment is essential to understand the effectiveness and performance of VANETs, simulation provides a few advantages over actual deployment Especially, the simulations are fast and repeatable and it is feasible to segregate the parameters such as real road map topology, vehicle movement, signal lights, and driving rules that affect the performance of VANETs. Segregation of parameters is crucial to understand the impact of each parameter on the VANETs. Moreover, simulations allow us to test the impact of parameters on VANETs, but it is complex or impossible in a real-world deployment.

As every vehicle is moving on a real road, one of the most significant properties of a mobile vehicle is the pattern of the vehicle's mobility [2]. It assists the VANETs to exploit the mobility models to describe the vehicle movement on the real road. In simulating VANETs, it is crucial to evaluate a realistic mobility model used in the VANETs, such that the observations from the simulation precisely pinpoints the actual road behavior. Choosing the appropriate value of parameters in the realistic model used in a VANETs simulation is a very complex process. However, there is a discrepancy between mobility patterns observable in reality and the derived theoretic mobility models for wireless networks [3] [4]. A realistic mobility model faithfully represents the unique dynamics of node mobility, characterized at a time by the real-time topologic maps that demonstrate the diverse road density and various classifications of the street with a miscellaneous speed limit of vehicles.

The conventional mobility models are classified into two types such as macroscopic, and microscopic. The macroscopic mobility model takes into account the traffic flow, described using parameters such as speed, density, and flow of vehicles. There remains an issue in the macroscopic model; it considers the traffic density as equal on the whole road map, but the traffic density is varied in different areas in a real-world environment. Moreover, the macroscopic mobility model cannot well adopt for real-time VANETs simulation. The microscopic mobility model shows the microscopic behavior of the vehicles by considering the traffic flow of a single vehicle as a basic unit, such as traveling speed, the distance between two vehicles, driver's acceleration, overtaking decisions, and realistic road topology changing parameters. However, the macroscopic mobility model requires significant memory space and high simulation time.

\section{EXISTING MOBILITY MODELS AND ITS} DRAWBACKS

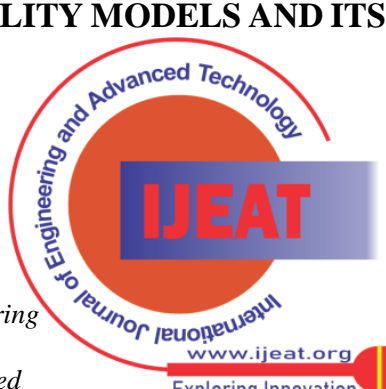


The mobility model represents the traces or patterns of vehicular nodes, position, velocity, and acceleration vary with time in the network.

The typical mobility models for wireless networks are random waypoints, random walks, random direction, reference points group, Manhattan, and obstacle model.

\section{A. Random Waypoint (RWP) Model:}

In RWP, the mobile node varies its position randomly without restrictions. The node selects a dynamic endpoint and a random velocity between zero and upper limit. This type involves the pause time between changes in destination and velocity. A major issue of RWP in the simulation is a poor choice of the velocity distribution.

\section{B. Random Walk (RW) model:}

In the RW model, the nodes are moved in random directions and speeds without pause time. The RW is a mobility model with forgetfulness property, in which the historical data of the earlier velocity is not taken into account for the future velocity. Thus, the RW of mobile nodes is not adequate for many real-life applications.

\section{Random Direction (RD) Model:}

The RD model enforces the vehicle to move to the corner of the surface in which the simulation is done beforehand the direction and the velocity of the vehicle is changed. The RD model fails to represent some mobility characteristics likely to exist in real roads such as obstacles, buildings, and streets. , the motion pattern in RD is unrealistic. Thus, it leads to produce incorrect results in routing protocol evaluation.

\section{Reference Point Group Model (RPGM):}

In RPGM, every node is the subset of the given cluster, and it follows a cluster head node that determines the group's motion behavior. The group member experiences random deviations from group motion. The main drawback of RPGM in the simulation is that node motion within a group is confined to relatively low-speed motion, but it is not suitable for high-speed VANETs scenarios.

\section{E. Manhattan model:}

This model utilizes a grid road topology, and the vehicle moves in the horizontal or vertical direction. It exploits a probability-based method in the choosing of node's movement at intersections. The Manhattan mobility model includes inter and intra-vehicle relationships. However, the Manhattan model considers a constrained environment that is not adequate for real-time applications.

\section{F. Obstacle model:}

This model includes obstacles like building in the real environment into account. In the obstacle model, the mobile nodes choose a proper movement trajectory to avoid running into such obstacles. However, the restricted movements of mobile nodes on the pathways are a major concern of the obstacle model.

Most of the conventional mobility models used in wireless networks do not consider real world factors like real road map topology and traffic rules and they can't be applied to VANETs as it is. The most important characteristic of a VANETs' mobility model is the degree of realism in the 


\section{B. Network Connectivity:}

The realistic mobility model is used to estimate the network connection parameters such as probability density functions (PDF) of node speed compared to another node and cumulative density functions (CDF) of the active period of the channel link with an average node degree. It is used to maintain better connectivity in VANETs simulation and effectively mimics the connectivity between vehicles in real-time.

\section{Street layouts:}

The roads force the vehicles to confine the movements of the vehicles in a pre-defined path. The realistic mobility models include constrained mobility pattern that governs the node spatial distributions and their interconnectivity. With a realistic mobility model, the drivers can take proper driving decisions using the connectivity information during driving.

\section{Traffic Control Mechanism:}

The VANETs can give data on the most usual traffic controlling mechanism at an intersection like stop signals and traffic light to the driver using a realistic model apart from the road map topologies.

\section{E. Inter-dependent Vehicular Motions:}

The particulars of the mobility pattern of the vehicle in a realistic mobility model instruct the drivers to keep a safe distance between the vehicles, vary their speed, and may change to adjacent lanes and thereby avoids the probability of accidents in VANETs.

\section{RELATED WORKS}

Several works attempt the design of the mobility model for VANETs. The papers [5] and [6] surveys the realistic mobility models used for VANETs. The work [7] attempts to understand the effect of the mobility model on the performances of VANETs. A comparative study in [8] discusses different VANETs mobility models with different tools. The conventional mobility models are classified into two types such as macroscopic, and microscopic [9].

\section{A. Macroscopic Mobility model}

A mobility model which has a dynamicity in the time domain has been proposed in [10] for the mobile wireless network. This model designs a realistic mobility model by employing a mobility trace generator that traces the mobility characteristics of average node degrees, hitting period, and meeting period of vehicles. A vehicular mobility framework in [11] does not consider the realistic parameters accurately such as real road topology, vehicle's priority, and speed limits in the mobility model design, whereas it considers the collision parameters into account. A macroscopic corridor model, METACOR has been proposed in [12], which is developed using two macroscopic traffic flows. The work [13] introduces a tool "MObility model generator for Vehicular Networks (MOVE)" that allows the user to produce realistic mobility models for VANETs simulations promptly. A group mobility model in [14], models the mobility of a cluster of mobile nodes. This model also includes individual mobile nodes and static nodes in the mobility model.
However, the macroscopic mobility model fails to deviate the traffic flow during rush hours. The Voronoi mobility model [15] takes into account the obstacles such as buildings and trees on the road in designing the mobility model. It can be simply applied to cities that are not too many complicated obstacles. However, the Voronoi model does not provide an optimal route to the vehicles.

\section{B. Microscopic Mobility Model:}

The work in [16], briefly describes the MOVE architecture. It includes the information about mobility models like the traffic signals information, path preference, and the vehicle overtaking behaviors having a non-trivial influence over the performance of VANETs simulation. Another work in [17] proposes two novel mobility models for VANETs. The first model is designed based on the vectored road map and the details about the speed limits are taken out from a geographical information system. The second model is designed as per the real-time traces of a microscopic multi agent traffic simulation system. A novel and realistic type of dynamic mobility model have been proposed in [18], in which the vehicular nodes have to slow down to the extent to the place where the direction of the vehicles change and speed up with a definite acceleration to attain its predetermined velocity.

A realistic mobility model in [19] adopts the social aspect of people mobility as well as the geographic limitations which govern the motion of the mobile vehicles. The work in [20] illustrates the influence of realistic mobility on the efficiency of the VANETs routing protocol. It investigates the significant parameters of the realistic mobility model. In [21], a vehicular mobility model is designed using two parameters. The first parameter is traffic information gathered by calculating the number of devices found at the Luxembourgian road networks. The next parameter is geographic data related to the diversified areas such as domestic, industrialized, and commercial areas. The work [22] presents a "VanetMobiSim," an open-source add-on to the "CanuMobiSim" user mobility platform, which can produce realistic node mobility patterns for VANETs simulation. A large-scale urban vehicular mobility trace model has been described in [23]. The model obtains the dataset by the keeping consideration of realistic road topologies, microscopic mobilities, and macroscopic flows. The work in [24] evaluates the spatial and temporal characteristics of VANETs using the realistic macroscopic and channel model. This model obtains the realistic large-scale mobility from accurate microscopic mobility modeling of SUMO that employs the node direction and velocity data as input parameters. Moreover, the microscopic mobility model requires high memory space and computational time.

The advantages and limitations of both the macroscopic and microscopic mobility models are given in Tables 1 and 2 .

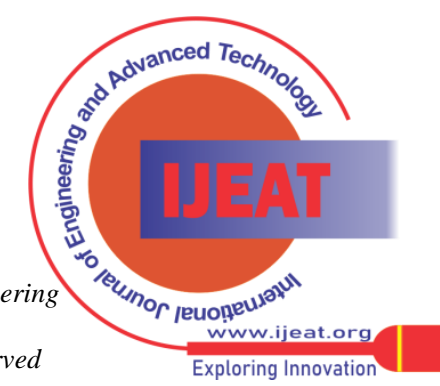


TABLE I. MACRO MOBILITY MODELS FOR VANETS

\begin{tabular}{|c|c|c|c|c|c|c|}
\hline \multirow{2}{*}{$\begin{array}{c}\text { Ma cro Mobility } \\
\text { Model }\end{array}$} & \multicolumn{4}{|c|}{ Macro Mobility Factors } & \multirow[b]{2}{*}{ Advantages } & \multirow[b]{2}{*}{ Limitations } \\
\hline & Multi-lane & $\begin{array}{l}\text { destination } \\
\text { position }\end{array}$ & Velocity & Acceleration & & \\
\hline $\begin{array}{l}\text { Time-variant } \\
\text { community mobility } \\
\text { Model }[10]\end{array}$ & No & Random & Road-dependent & No & $\begin{array}{c}\text { Flexible for mobility } \\
\text { traces over different } \\
\text { scenarios }\end{array}$ & $\begin{array}{l}\text { Consi dering } \\
\text { constant traffic is } \\
\text { inadequate for } \\
\text { different areas }\end{array}$ \\
\hline MCRV [11] & Yes & Random & Road-dependent & yes & $\begin{array}{l}\text { Enables a fast and low } \\
\text { cost accessability of data } \\
\text { for the use of driver's } \\
\text { wellbeing and } \\
\text { comfortness }\end{array}$ & $\begin{array}{c}\text { Fails to deviate the } \\
\text { traffic flow in rush } \\
\text { hours }\end{array}$ \\
\hline $\begin{array}{l}\text { An integrated } \\
\text { Simulator [13] }\end{array}$ & Yes & Random & Driver- dependent & Yes & $\begin{array}{l}\text { It includes the lane } \\
\text { changing model and } \\
\text { improves road safety }\end{array}$ & $\begin{array}{l}\text { Improper dynamic } \\
\text { driver decisions } \\
\text { affect the protocol } \\
\text { perf ormance }\end{array}$ \\
\hline Virtual Track [14] & No & Random & Uniform & No & $\begin{array}{c}\text { It models the dynamics of } \\
\text { group m obility that } \\
\text { avoids road accidents }\end{array}$ & $\begin{array}{l}\text { It cannot adapt to } \\
\text { different real-time } \\
\text { VANETs } \\
\text { scenarios } \\
\end{array}$ \\
\hline Voronoi model [15] & No & Random & Uniform & No & $\begin{array}{l}\text { The obstacle detection } \\
\text { increases road safety }\end{array}$ & $\begin{array}{l}\text { Cannot be applied } \\
\text { to the cities where } \\
\text { there are too many } \\
\text { complicated } \\
\text { obstacles }\end{array}$ \\
\hline
\end{tabular}

TABLE II. MICRO MOBILITY MODELS FOR VANETS

\begin{tabular}{|c|c|c|c|c|}
\hline $\begin{array}{c}\text { Micro Mobility } \\
\text { Model }\end{array}$ & Micro Mobility Factors & Topology & Advantages & Limitations \\
\hline MOVE [16] & $\begin{array}{l}\text { Human patterns, Real Road } \\
\text { map }\end{array}$ & Urban and Rural & $\begin{array}{l}\text { The vehicles can } \\
\text { dynamically adjust the } \\
\text { routes during simulation }\end{array}$ & $\begin{array}{l}\text { Simulation results might } \\
\text { not be closer to real time }\end{array}$ \\
\hline $\begin{array}{l}\text { Novel mobility } \\
\text { models [17] }\end{array}$ & $\begin{array}{l}\text { Human patterns, Real Road } \\
\text { map, Lane Changing }\end{array}$ & Urban & $\begin{array}{l}\text { It provides a higher level of } \\
\text { details }\end{array}$ & $\begin{array}{l}\text { Very complex to } \\
\text { implement in real time }\end{array}$ \\
\hline $\begin{array}{c}\text { Realistic } \\
\text { mobility model } \\
{[18]}\end{array}$ & $\begin{array}{l}\text { Human patterns, Real Road } \\
\text { map, Lane Changing }\end{array}$ & Urban & $\begin{array}{l}\text { Finds every possible } \\
\text { correlation am ong the } \\
\text { accel erated movem ents of } \\
\text { the mobile nodes }\end{array}$ & $\begin{array}{c}\text { Inaccurate measurem ent } \\
\text { of direction changes }\end{array}$ \\
\hline $\begin{array}{c}\text { Geo-social } \\
\text { mobility model } \\
{[19]}\end{array}$ & $\begin{array}{c}\text { Human patterns, Real Road } \\
\text { map, Obstacles }\end{array}$ & Urban and Rural & $\begin{array}{l}\text { It integrates both the social } \\
\text { aspect of people } \\
\text { mobilities with the } \\
\text { geographic restriction which } \\
\text { manage the movem ents of } \\
\text { the } \\
\text { mobile node }\end{array}$ & $\begin{array}{l}\text { Requires highmem ory } \\
\text { space }\end{array}$ \\
\hline VehILux [21] & Real Roadmap & Urban & $\begin{array}{l}\text { This model could produce a } \\
\text { reali stic mobility model for } \\
\text { any areas, for the traffic } \\
\text { count data present }\end{array}$ & $\begin{array}{l}\text { It does not address the } \\
\text { values of its param eters } \\
\text { should be set }\end{array}$ \\
\hline $\begin{array}{c}\text { Mobility } \\
\text { framework [22] }\end{array}$ & $\begin{array}{c}\text { Human patterns, Real Road } \\
\text { map, L ane Changing. } \\
\text { Obstacles }\end{array}$ & Urban & $\begin{array}{l}\text { Provides a freely available } \\
\text { generator of realistic } \\
\text { vehicular movem ent traces }\end{array}$ & $\begin{array}{l}\text { Inaccurate attraction and } \\
\text { repulsion points of } \\
\text { parameters create a } \\
\text { bottleneck in the } \\
\text { simulation }\end{array}$ \\
\hline
\end{tabular}




\section{PROBLEM STATEMENT}

Several realistic mobility models have been designed for improving the accuracy of VANETs simulation. The quality of VANETs simulations is based on the degree of realities of the mobility models. Several vehicular mobility models, allow VANETs to generate realistic mobility traces by utilizing the real traffic density data that refers to the counts of the vehicle along a given road. The real traffic volume count offers accurate data about the density of vehicles and their type. For generating the real traffic count data, most of the existing works rely on probabilistic geographical attraction point that includes the preferred roads depending on the driver's decision in reaching the destination of the vehicle.

However, the existing works do not attempt to elect the effective value of the probability of selecting a preferred road to reach the destination each route is allotted with the probability of being selected as the best one. It is affiliated with the attraction points, as the values depend on the real-time instances of VANETs. It is crucial to find out the best probability values of attraction points to improve the mobility model realism. Also, it is essential to take into account the driver's specific behaviors such as lane changing and overtaking decisions in rush hours to improve the probabilistic point detection accuracy.

\section{GAP ANALYSIS}

The pattern in which the node moves in an area of interest is a key point in VANETs simulation. The real-world traces of individual vehicles and realistic road map topology are the most precise representation of the mobility models. The microscopic mobility models are desirable for a real-time VANETs simulation as it considers the drivers' behavior and real-time road topology in the design of the mobility model. In the real-world environment, the nodes should dynamically alter their paths as per various traffic conditions with different dynamic factors such as accidents. The microscopic mobility model fails to address the accurate selection of real traffic data, as it does not estimate the probability of dynamic factors accurately. Moreover, the performance of VANETs simulation is poor in realistic transportation simulation environments. The prediction model estimates the overall performance of around $80 \%$ [25]. It is very effective to use realistic mobility models that exploit accurate, realistic traffic dynamic factors to exhibits the real-time performance of a VANETs. The proposed work aims at improving the detection accuracy of attraction points and designing an optimal realistic mobility model for real-time VANETs simulation.

\section{PROPOSED METHODOLOGY 1}

\section{A. Aim and Objectives:}

- To generate the realistic mobility models to the actual road map topologies, the proposed work designs a mobility model, REAL-VM for VANETs.

- To mimic the actual motion of vehicles, the REAL-VM includes the real road map topology and attractivity factors for generating mobility patterns.
- To react to the changes in road conditions such as traffic jams and lane changing parameters, and to alert the drivers during accidents.

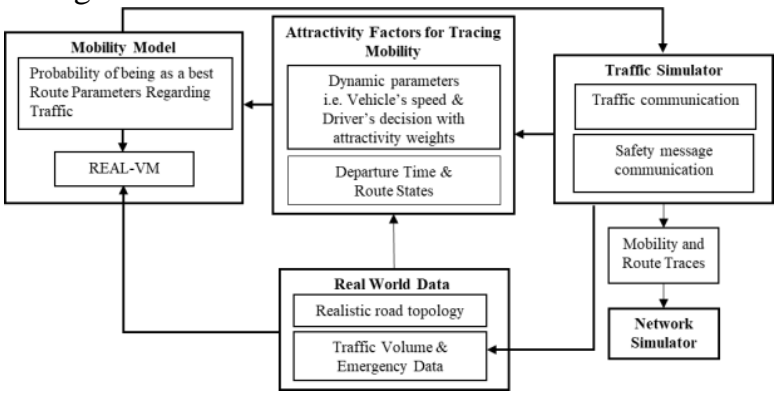

Fig. 1. Architecture of REAL-VM

This work proposes a REAListic Vehicular Mobility (REAL-VM) model that includes realistic road topology with attractivity factors for producing the mobility model and improve the accuracy of simulation results of VANETs. The architecture of the proposed work is detailed in Figure 1. This model employs two types of data as inputs that describe the real-world characteristics such as real-world data and attractivity factors for generating the mobility pattern. The real-world data include a realistic road map and the traffic density in the area covered. At the initialization stage, the proposed work designs the mobility model by considering only the real-world data. To improve the mobility model, dynamic attractivity parameters are measured during the vehicle movement and included in the mobility model.

The attractivity factors include the dynamic parameters such as the vehicle's speed, driver's decision, departure time, and route states because these are the main factors to generate the mobility pattern. The Dynamic Clustering Algorithm (DCA) for VANETs mentioned in [26] stabilize the clusters and minimize the cluster re-affiliation period. The speed of the vehicle is designed using real-time factors such as accidents and traffic jams. The main function of electing the group head node is done depends on vehicle mobility parameters including location, speed, and packet forwarding capacity, the algorithm of the clustering [27]. The driver's decision refers to the preferred roads to go to the place where he wants to go. The departure time is the approximate time at which the vehicle is estimated to leave the source point. To measure these factors, consider the real road map topology has $n$ states over the area, $\mathrm{A}(\mathrm{Ai}=\mathrm{A} 1 \ldots \mathrm{An})$. The routing state refers to the probability of a vehicle entering into ' $n$ ' state from ' $m$ ' state. The attractivity factors for generating mobility patterns are measured using the traffic simulator, i.e., simulation of urban mobility (SUMO). The traffic simulator generates the original data of VANETs by using the communication of traffic and safety messages. This traffic volume is given as input to the measurement of attractive factors. The network simulator exploits the mobility and traffic factors to simulate the VANETs.

Blue Eyes Intelligence Engineering

\& Sciences Publication 


\section{PROPOSED METHODOLOGY 2}

\section{A. Aim and Objectives:}

- To investigate the impact of human driver decision and vehicle speed on a realistic mobility model in VANETs.

- To mimic the real motion of vehicles, by considering the presence of traffic signals, driver route preference, and vehicle overtaking behaviors.

- To improve the performance of the mobility model and to tightly integrate the traffic and network simulator with a mobility model.

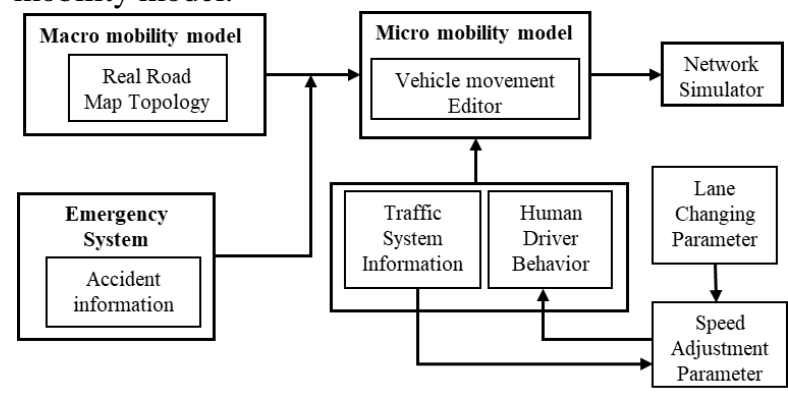

Fig. 2. Architecture of REVEAL

This paper proposes a REalistic moVEment pAttern of the vehicLes (REVEAL) model to improve the real-time performance of VANETs simulation. The block diagram of REVEAL consists of three main parts named a macro mobility model, micro-mobility model, and emergency system.

The macro mobility model includes the realistic road map topology designed by the users or derived automatically or extracted from existing real-world maps. The micro-mobility model has a component named as vehicle movement editor. The traffic system information and the human driver behavior are the inputs of the vehicle movement editor. PDR and the node to node delay are studied with the help of AOMDV in different traffic environments and simulation parameters [28]. The traffic system information contains two types of information such as traffic management, parking space management, and traffic jam avoidance. The nodes are commanded to cease in the traffic signal to pause for the signal till green. Moreover, the human driver behavior creates a significant impact on the mobility model, and the proposed REVEAL considers the complex behavior models of a driver like speed adjustment parameter.

WECA-MR is proven to be an efficient clustering algorithm and as an efficient tactic to enhance considerably the performance of the wireless vehicle networks. To select the Cluster Heads (CHs), classic weight metrics are employed. Few of such metrics as degree, mean distance, and a new parameter presented by a scheme known as Mobility Report (MR) [29]. The REVEAL considers the impact of change in road curvature and the node longitudinal and directional speed on a curved road in designing the mobility model.

Another important factor to consider in the vehicle movement pattern is the emergency system, including an emergency event like an accident. Mapping different aspects of mobility models including road map topology, and human driver individual and neighbors' decision, the manner of decision-making at lane changing, and the adjustment of a vehicle's speed lead to developing the model more realistic. Routing Protocol for Low power lossy network (RPL) is a very efficient routing protocol in IoT that can be employed in VANETs but it has problems related to mobility [30].

\section{EXPERIMENTAL REQUIREMENTS}

\section{A. Simulator:}

- Network Simulator-2.35

This is a tool for reproducing real-time networking scenarios and their operating and related standards respectively with the help of simulating it.

\section{B. Tools:}

- Network Animator (NAM)-1.15

NAM is an animation tool that depends on Tcl/TK that helps view the network simulation patterns and the real-time packet patterns [31]. It provides the facility to draw topology, level packet animation, and utilities for various data inspections. - Xgraph-12.2

XGRAPH is a generic Cartesian trend plotting tool with the user interface to navigate, print, and select the option given in the display. Data points are plotted from various files in the single Cartesian plane and it can support handling n number of information-set size and n number of files.

- Simulation of Urban Mobility (SUMO)-0.12.3

It is a tool to simulate low level traffic conditions where every nodes are designed in an explicit manner.

\section{Languages:}

- Tool Command Language (TCL) and Otcl-1.14

The TCL is a scripting language, and the Otcl refers to an object-oriented extension of TCL.

- AWK script

The AWK is an interpreted programming language specifically used to process text and generally used for extract and report data.

- $\mathrm{C}++$

It is a general purpose object-oriented programming language. It provides low-level memory manipulation as well.

\section{Road Map and Mobility Generator:}

- REAL-VM

- REVEAL

\section{E. Performance Metrics:}

The proposed REAL-VM and REVEAL consider some parameters such as PDR, node to node delay, and Road Safety Assurance.

\section{F. Packet Delivery Ratio (PDR):}

PDR is given by the ratio of the number of information packets productively reached destination to a no. of the packet transmitted by the sender.

\section{G. Delay:}

This parameter is the average period elapsed for a data set to travel through the network from one state to the destination state.

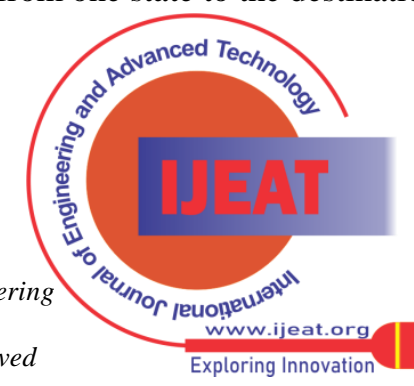
Exploring Innovation
Published By:

Blue Eyes Intelligence Engineering

\& Sciences Publication

(C) Copyright: All Rights Reserved 


\section{H. Throughput:}

It is a quantity that gives the amount of data delivered successfully at the destination.

\section{Road Safety Assurance:}

It is the ratio of delivering road safety messages within a period.

\section{J. Relative Mobility:}

It is defined as the average changes in distances between two vehicles.

\section{K. Tracking Error:}

It is a vectored quantity from the predictive reference vector and the controlled vehicle traveling vector.

\section{CONCLUSION}

To precisely pinpoint the real road performance, this work proposes two different realistic mobility models for VANETs. Choosing the appropriate value of parameters in the realistic traffic model, the REAL-VM reduces the discrepancy between mobility patterns observable in reality and the derived theoretic mobility models for wireless networks. By reflecting the diverse road densities and different groups of the street with a miscellaneous speed limit of vehicles, the proposed mobility model, REVEAL faithfully represents the unique dynamics of node mobility. The speed of the vehicle is adjusted based on the road and lane changing decisions of neighboring vehicles, traffic system information, and movement pattern of neighboring vehicles. Because the faster drivers take over taking decisions by considering the neighboring vehicle's lane changing decisions. Mobility Prediction Based Routing Protocol gives PDR of about 26\% by the average in grid-based scenarios, and about $\sim 26 \%$ by the average in urban scenarios respectively.

\section{REFERENCES}

1. S. Zeadally, R. Hunt, Y. S. Chen, A. Irwin, and Hassan, "A Vehicular ad hoc networks (VANETS): status, results, and challenge," Telecommunication Systems, vol. 50, No. 4, pp. 217-24, 2012

2. F. K. Karnadi, Z. H. Mo, and K.-C. Lan, "Rapid generation of realistic mobility models for VANET," in Proc. IEEE WCNC, pp. 2506-2511, 2007

3. M. M. Zonoozi and P. Dassanayake, "User mobility modeling and characterization of mobility patterns," IEEE J. Sel. Areas Commun, vol. 15, No. 7, pp. 1239-1252, 1997

4. F. Ashtiani, J. A. Salehi, and M. R. Aref, "Mobility modeling and analytical solution for spatial traffic distribution in wireless multimedia networks," IEEE J. Sel. Areas Communication, vol. 21, No. 10, pp. 1699-1709, 2003

5. Djenouri, Djamel, Elmalik Nekka, and Wassim Soualhi, "Simulation of mobility models in vehicular ad hoc networks," In Proceedings of the Ambi-Sys workshop on Software Organisation and MonIToring of Ambient Systems ICST (Institute for Computer Sciences, Social-Informatics and Telecommunications Engineering), p. 4, 2008

6. S. Madi and H. Al-Qamzi, "A survey on realistic mobility models for vehicular ad hoc networks (VANETs)", In Networking, Sensing and Control (ICNSC) 10th IEEE International Conference, pp. 333-339, 2013

7. Mahajan, Atulya, Niranjan Potnis, Kartik Gopalan, and Andy Wang, "Urban mobility models for vanets," In 2nd IEEE International Workshop on Next Generation Wireless Networks, vol. 33, 2006

8. D. Djenouri, W. Soualhi, and E. Nekka, "VANET's mobility models and overtaking: an overview," In Information and Communication Technologies: From Theory to Applications 3rd International Conference IEEE, pp. 1-6, 2008
9. Helbing Dirk, "Traffic and related self-driven many-particle systems," Reviews of modern physics, vol. 73, No. 4, pp. 1067, 2001

10. Hsu, Wei-Jen, Thrasyvoulos Spyropoulos, Konstantinos Psounis, and Ahmed Helmy, "Modeling spatial and temporal dependencies of user mobility in wireless mobile networks," IEEE/ACM Transactions on Networking (TON), vol. 17, No. 5, pp. 1564-1577, 2009

11. P. Vetrivelan, P. Narayanasamy, and JC John Charlas, "A Multi-Constraint Real-time Vehicular (MCRV) Mobility Framework for 4G Heterogeneous Vehicular Ad-Hoc Networks," In Proceedings of the International MultiConference of Engineers and Computer Scientists, vol. 1. 2012

12. Salem, H. Haj, J. Chrisoulakis, M. Papageorgiou, N. Elloumi, and P. Papadakos, "The use of METACOR tool for integrated urban and interurban traffic control evaluation in corridor peripherique, Paris," In Vehicle Navigation and Information Systems Conference, Proceedings, pp. 645-650, 1994

13. Gorgorin, Cristian, Victor Gradinescu, Raluca Diaconescu, Valentin Cristea, and L. Ifode, "An integrated vehicular and network simulator for vehicular ad-hoc networks," In Proceedings of the European Simulation and Modelling Conference (ESM), Bonn, Germany, 2006

14. Zhou, Biao, Kaixin $\mathrm{Xu}$, and Mario Gerla, "Group and swarm mobility models for ad hoc network scenarios using virtual tracks," In Military Communications Conference IEEE, vol. 1, pp. 289-294, 2004

15. Zimmermann, Hans-Martin, Ingo Gruber, and Christian Roman, "A Voronoi-based mobility model for urban environments," In Wireless Conference Next Generation Wireless and Mobile Communications and Services (European Wireless), 11th European, pp. 1-5, 2005

16. Lan, Kun-chan, and Chien-Ming Chou, "Realistic mobility models for vehicular ad hoc network (VANET) simulations," ITS Telecommunications ITST 8th International Conference IEEE, 2008

17. Wu, Aifeng, Jianqing $\mathrm{Ma}$, and Shiyong Zhang, "Towards realistic mobility modeling for vehicular ad hoc networks," In Proceedings of the 44th Annual Simulation Symposium Society for Computer Simulation International, pp. 57-67, 2011

18. Zarifneshat, Masoud, and Pejman Khadivi, "Using mobile node speed changes for movement direction change prediction in a realistic category of mobility models," Journal of Network and Computer Applications, vol. 36, No. 3, pp. 1078-1090, 2013

19. Basta, Nardine, Amal El-Nahas, Hans-Peter Grossmann, and Slim Abdennadher, "Geo-social mobility model for VANET simulation," Journal of Mobile Multimedia, vol. 10, No.2, pp. 107-127, 2014

20. Härri, Jérôme, Fethi Filali, and Christian Bonnet, "On meaningful parameters for routing in vanets urban environments under realistic mobility patterns," In Process of the IEEE Workshop on Automotive Networking and Applications (AutoNet)(in conjunction with IEEE GLOBECOM), 2006

21. Pigné, Yoann, Grégoire Danoy, and Pascal Bouvry, "A vehicular mobility model based on real traffic counting data," In Communication Technologies for Vehicles Springer Berlin Heidelberg, pp. 131-142, 2011

22. Härri, Jérôme, Marco Fiore, Fethi Filali, and Christian Bonnet, "Vehicular mobility simulation with VanetMobiSim," Simulation, 2009

23. Uppoor, Sandesh, and Marco Fiore, "Large-scale urban vehicular mobility for networking research," In Vehicular Networking Conference (VNC) IEEE, pp. 62-69, 2011

24. Akhtar, Naheed, Sinem Coleri Ergen, and Oznur Ozkasap, "Vehicle mobility and communication channel models for realistic and efficient highway VANET simulation," Vehicular Technology IEEE Transactions, vol. 64, No. 1, pp. 248-262, 2015

25. Wei Fan, Yan Shi, Shanzhi Chen and Longhao Zou, "A mobility metrics based dynamic clustering algorithm for VANETs," IET International Conference on Communication Technology and Application (ICCTA 2011), Beijing, 2011, pp. 752-756, doi: 10.1049/cp.2011.0769.

26. G. J. Anaya-López, C. Cárdenas-Angelat, D. Jiménez-Soria, M. C. Aguayo-Torres, N. Guerra-Melgares and J. Baños-Polglase, "Quality of experience prediction in mobility scenarios based on recurrent neural networks," 2020 IEEE 91st Vehicular Technology Conference (VTC2020-Spring), Antwerp, Belgium, 2020, pp. 1-5, doi: 10.1109/VTC2020-Spring48590.2020.9128690.

27. X. Ge, Q. Gao and X. Quan, "A Novel Clustering Algorithm Based on Mobility for VANET," 2018 IEEE 18th International Conference on Communication Technology (ICCT), Chongqing, 2018, pp. 473-477, doi: 10.1109/ICCT.2018.8600156.

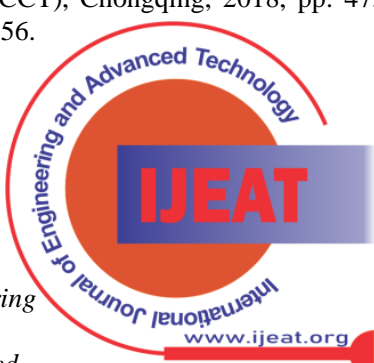

Blue Eyes Intelligence Engineering 
28. A. T. Sasongko, G. Jati and W. Jatmiko, "Multipath Routing Protocols in Vehicular Ad hoc Networks (VANETs): Performance Evaluation in Various Conditions," 2018 3rd International Seminar on Sensors, Instrumentation, Measurement and Metrology (ISSIMM), Depok, Indonesia, 2018, pp. 74-78, doi: 10.1109/ISSIMM.2018.8727733.

29. O. Senouci, S. Harous and Z. Aliouat, "An Efficient Weight-Based Clustering Algorithm using Mobility Report for IoV," 2018 9th IEEE Annual Ubiquitous Computing, Electronics \& Mobile Communication Conference (UEMCON), New York City, NY, USA, 2018, pp. 614-620, doi: 10.1109/UEMCON.2018.8796619.

30. Y. Tang, N. Cheng, W. Wu, M. Wang, Y. Dai and X. Shen, "Delay-Minimization Routing for Heterogeneous VANETs With Machine Learning Based Mobility Prediction," in IEEE Transactions on Vehicular Technology, vol. 68, no. 4, pp. 3967-3979, April 2019, doi: 10.1109/TVT.2019.2899627.

31. C. Celes, F. A. Silva, A. Boukerche, R. M. d. C. Andrade and A. A. F. Loureiro, "Improving VANET Simulation with Calibrated Vehicular Mobility Traces," in IEEE Transactions on Mobile Computing, vol. 16, no. 12, pp. 3376-3389, 1 Dec. 2017, doi: 10.1109/TMC.2017.2690636.

\section{AUTHORS PROFILE}

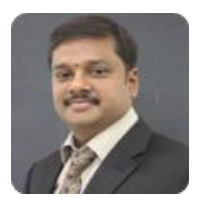

V. Hariharasudhan, received a Bachelor's degree in Electronics and Instrumentation Engineering and MBA degrees from Bharathiar University, Coimbatore, and MTech in Embedded Systems and Technology from SRM University, Chennai. Currently, he is working as a Senior Engineering Manager in Johnson Controls, Pune. He has 22 years of rich experience in end to end product design \& development of embedded system controls in the measurement, automotive domain \& Building Automation Technology. In Johnson's Controls, he is heading the Embedded Controls team for various BUs of JCI's Controls Product Development group in India Engineering Center. Leading a team of 130 members of various functions like Hardware design \& development, Firmware, User Interface, Mobility, Cloud, IoT, DevOps, Cyber Security, Controls apps using MATLAB, Industrialization (MCAD, ECN/FCN/PLM), Mechanical design \& development, Controls lab, prototyping, manufacturing \& sustaining, Test automation using Labview, Teardown and benchmarking. Responsible for Engineering management, Delivery management, Project management, Innovation and Process compliance. Managing multiple projects and working with global engineering centers. He has strong experience and concepts in designing and developing Automotive Instrument clusters, Electronic control units, Body Control modules, Power Train and Telematics and HVAC control products. He worked in 3 startup companies/teams, built the team and infrastructure from the scratch. His research interests are Wireless sensor networking, VANETs mobility, Embedded Controls, Cloud computing, Data Science, and IoT.

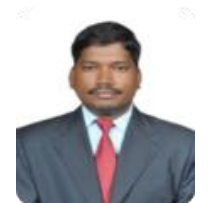

Dr. Vetrivelan P, Ph.D., is an Associate Professor Senior and Head of the Department (HoD)/Programme Chair for Bachelor of Technology (Electronics and Communication Engineering) in School of Electronics Engineering at Vellore Institute of Technology (VIT), Chennai, India. He has completed a Bachelor of Engineering from the University of Madras, Chennai, and both Master of Engineering in Embedded Systems Technologies and Doctor of Philosophy in Information and Communication Engineering from Anna University, Chennai. He has 16.1 rich years of teaching experience altogether in CSE and ECE Departments in both private Engineering Colleges in Chennai (affiliated to Anna University, Chennai) and Private Engineering University in Chennai respectively. He has authored 5 book chapters and one proceeding in lecture notes published by a reputed springer publisher and has authored 32+ Scopus indexed Journal papers and few other papers published in reputed international conferences. He has served as a member of the Board of Studies, doctoral committee, doctoral thesis Examiner, doctoral oral Examiner in both private and government Universities. He has also served as a reviewer for reputed International Journals and International Conferences. His research interests include Wireless Networks, Adhoc and Sensor Networks, Vehicular ad hoc networks (VANETs), Embedded Systems, and Internet of Things (IoT) with Machine Learning.

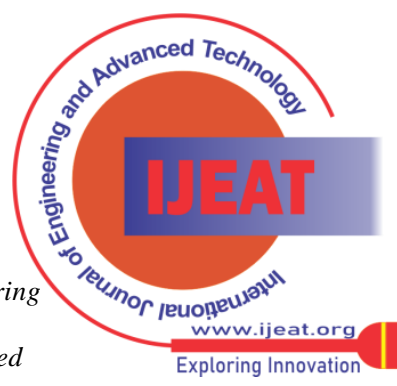

